\title{
IEEE Transactions on Wireless Communications
}

- accepted for publication -

\section{Nearly Doubling the Throughput of Multiuser MIMO Systems Using Codebook Tailored Limited Feedback Protocol}

\author{
GERhard Wunder ${ }^{1}$, JAN SCHRECK ${ }^{2}$ AND PETER JunG ${ }^{2}$
}

${ }^{1}$ Fraunhofer Heinrich Hertz Institute, Einsteinufer 37, D-10587 Berlin, Germany

${ }^{2}$ Technische Universität Berlin Lehrstuhl für Informationstheorie und Theoretische Informationstechnik, Einsteinufer 25, D-10587 Berlin, Germany

October 19, 2018

(C)2012 IEEE. Personal use of this material is permitted. Permission from IEEE must be obtained for all other uses, in any current or future media, including reprinting/republishing this material for advertising or promotional purposes, creating new collective works, for resale or redistribution to servers or lists, or reuse of any copyrighted component of this work in other works. 


\begin{abstract}
We present and analyze a new robust feedback and transmit strategy for multiuser MIMO downlink communication systems, termed Rate Approximation (RA). RA combines the flexibility and robustness needed for reliable communications with the user terminal under a limited feedback constraint. It responds to two important observations. First, it is not so significant to approximate the channel but rather the rate, such that the optimal scheduling decision can be mimicked at the base station. Second, a fixed transmit codebook at the transmitter is often better when therefore the channel state information is more accurate. In the RA scheme the transmit and feedback codebook are separated and user rates are delivered to the base station subject to a controlled uniform error. The scheme is analyzed and proved to have better performance below a certain interference plus noise margin and better behavior than the classical Jindal formula. LTE system simulations sustain the analytic results showing performance gains of up to $50 \%$ or $70 \%$ compared to zeroforcing when using multiple antennas at the base station and multiple antennas or a single antenna at the terminals, respectively. A new feedback protocol is developed which inherently considers the transmit codebook and which is able to deal with the complexity issue at the terminal.
\end{abstract}

\title{
I. INTRODUCTION
}

Multiuser multiple input multiple output (MU-MIMO) communication systems have been in the focus of intensive research over many years. The optimal transmission technique for these systems is dirty paper coding (DPC), which, under perfect channel state information at the transmitter (CSIT), achieves superior performance gains over linear schemes. However, in practical systems CSIT is obtained via a rate-constrained feedback channel, which is known to be a sensitive part of the overall system and must be carefully designed.

In this paper we revisit the limited feedback problem in MU-MIMO systems. We consider linear beamforming and assume that the transmit beamforming vectors are defined by a fixed transmit codebook known to the base stations and all users. In contrast to previous work, we use a different codebook for the feedback and apply a new feedback strategy which we call Rate Approximation (RA). Loosely speaking, using the proposed RA feedback strategy, the terminal selects a channel quantization vector from the feedback codebook considering any possible scheduling decision that can be taken by the base station. As we show, this will enable the base station to approximate the user rates (rather than the user channels) subject to a small uniform a priori error. Then, given the feedback message, the base station is permitted to assert any beamforming vector from the transmit codebook for some network oriented optimization purpose (not just the beamforming vector dictated by the user).

\section{A. Related Work}

An extensive survey on limited feedback in wireless multiple antennas systems can be found in [1]. The standard reference for point-to-point multiple input single output (MISO) systems is [2] where groundbreaking analytical expressions for the problem are derived. Reference [3] evaluates the performance of point-to-point MISO systems using random vector quantization (RVQ). In [4] is shown that RVQ is asymptotically optimal for point-to-point MIMO systems.

For MU-MIMO systems, which are in the focus of this paper, reference [5] provides the standard performance analysis for the throughput degradation assuming RVQ. In [6] different feedback schemes are proposed that enable the base station to estimate the signal-to-noise-plus-interference ration (SINR) of each user. However, both papers specifically assume zeroforcing (ZF) beamforming and no individual user rate analysis is provided. Reference [7] also considers ZF and jointly designs the receive filters and the channel quantization to maximize the expected SINR of each user. In [8] different kinds of partial CSIT are assumed and the performance of DPC and ZF is compared.

Another popular transmission technique is unitary beamforming (UB). UB with a sum feedback rate constraint is considered in [9]. In [10] a UB scheme named per user unitary rate control (PU2RC) has been proposed for LTE. In [11] an improved user selection scheme for PU2RC is proposed. Again, no error analysis for individual user rates has been presented.

In contrast to previous work, this paper considers an arbitrary transmission scheme for a fixed transmit codebook and analyzes the individual rate error.

\section{B. Organization and Main Results}

In Section II we introduce the system model and in Section III the RA scheme is introduced. In Section IV.

1) We analyze the a priori rate error at the base station (before any scheduling decision) for each individual terminal evoked by our RA feedback strategy. We prove that it has better scaling properties compared to the classical result in [5] and that this benefit improves with an increasing number of transmit antennas.

2) We outline an advanced vector quantization problem related to the RA scheme by replacing the common chordal distance with a new distance function which inherently uses the structure of the transmit codebook.

In Section $\nabla$ we underline our results with LTE system simulations showing the benefit obtained by the proposed RA scheme and develop a suboptimal feedback protocol dealing with the complexity issue. This feedback protocol is proposed to replace the common approach for LTE. Finally, in Sec. VI the conclusion is drawn with emphasis on the impact on future standards.

Notation: Bold letters denote vectors and bold capital letters matrices. The inner product between vectors $\boldsymbol{a}$ and $\boldsymbol{b}$ is defined as $\langle\boldsymbol{a}, \boldsymbol{b}\rangle=\boldsymbol{a}^{H} \boldsymbol{b}$, where $\boldsymbol{a}^{H}$ is the conjugate transpose of the vector $\boldsymbol{a}$. The (euclidean) $\ell_{2}$-norm is $\|\boldsymbol{a}\|_{2}:=\langle\boldsymbol{a}, \boldsymbol{a}\rangle^{1 / 2}$. $\mathbb{S}^{n-1}$ is the unit sphere in $\mathbb{C}^{n}$. The $\ell_{1}$-norm of a vector $\boldsymbol{a}$ with components $a_{j}$ is defined as $\|\boldsymbol{a}\|_{1}:=\sum_{j}\left|a_{j}\right|$ and $\|\boldsymbol{a}\|_{\infty}:=\max _{j}\left|a_{j}\right|$ denotes its $\ell_{\infty}-$ norm. 


\section{SySTEM SETUP}

We consider the MU-MIMO downlink channel of a cellular system where a base station, equipped with $n_{t}$ transmit antennas, serves multiple users, equipped with $n_{r}$ receive antennas, on the same time and frequency resource with a single data stream. The users are collected in the set $\mathcal{U}$. Let $\boldsymbol{x} \in \mathbb{C}^{n_{t}}$ be the signal transmitted by the base station in a single transmission interval (time index omitted). User $m$ receives the transmitted signal through the channel $\boldsymbol{H}_{m} \in \mathbb{C}^{n_{r} \times n_{t}}$ and applies a fixed receive filter $\boldsymbol{u}_{m} \in \mathbb{C}^{n_{r}}$ to recover its intended signal,

$$
y_{m}=\left\langle\boldsymbol{u}_{m}, \boldsymbol{H}_{m} \boldsymbol{x}\right\rangle+n_{m}=:\left\langle\hat{\boldsymbol{h}}_{m}, \boldsymbol{x}\right\rangle+n_{m}
$$

where $n_{m} \sim \mathcal{C N}\left(0, \sigma^{2}\right)$ is additive white Gaussian noise (zero-mean with variance $\sigma^{2}$ ) and $\hat{h}$ is the effective channel vector from the base station to user $m$. In the sequel we assume each user $m$ has perfect knowledge of its own channel $\boldsymbol{H}_{m}$ and that the channels are constant over one transmission interval; no fading model is imposed. Moreover, we assume no delay in the CSIT report, scheduling or transmission.

In MU-MIMO systems adaptive adjustment of the number of active users is crucial to achieve high spectral efficiency, see [12] and references therein. In each transmission interval the base station selects a subset $\mathcal{S} \subseteq \mathcal{U}$ of users for transmission on the same spectral resource and assigns each user $m \in \mathcal{S}$ a beamforming vector out of a finite transmit codebook $\mathcal{C} \subset \mathbb{S}^{n_{t}-1}$, known to the base station and all users. We will denote with $[\mathcal{C}]:=[1 \ldots|\mathcal{C}|]$ the set of codeword indices. The assignment of users to beamforming vectors is defined by a mapping

$$
\pi: \mathcal{S} \rightarrow[\mathcal{C}]
$$

that maps each element $m \in \mathcal{S}$ to a codebook element $\boldsymbol{w}_{\pi(m)} \in \mathcal{C}$. We assume that $|\mathcal{S}| \leq n_{s} \leq n_{t}$, where $n_{s}$ is the maximum number of users that can be scheduled on a spectral resource. Note that we do not state the domain of $\pi$ explicitly, if it is clear from the context. In the sequel we may assume that the codebook has the property 1 that for every $f \in \mathbb{C}^{n_{t}}$

$$
\sum_{\boldsymbol{w} \in \mathcal{C}}|\langle\boldsymbol{w}, \boldsymbol{f}\rangle|^{2}=A\|\boldsymbol{f}\|_{2}^{2},
$$

with a fixed constant $A \geq 1$. If $A=1, \mathcal{C}$ constitutes an orthonormal base (ONB) and we call $\mathcal{C}$ an unitary codebook (used for UB).

Define the complex information symbols intended for user $m$ as $d_{m} \in \mathbb{C}$, the transmitted signal is given by the superposition

$$
\boldsymbol{x}=\sqrt{\frac{P}{|\mathcal{S}|}} \cdot \sum_{m \in \mathcal{S}} \boldsymbol{w}_{\pi(m)} d_{m},
$$

where we assumed equal power allocation with the power budget $P$. The achieved sum rate for some user set $\mathcal{S}$ and mapping $\pi$ is

$$
R(\pi, \mathcal{S}, H)=\sum_{m \in \mathcal{S}} r_{m}\left(\pi, \mathcal{S}, \hat{\boldsymbol{h}}_{m}\right)
$$

where $H=\left\{\hat{\boldsymbol{h}}_{m}\right\}_{m \in \mathcal{U}}$ is the list of effective channels. The per user contributions to the sum rate are given by the Shannon rates

$$
r_{m}\left(\pi, \mathcal{S}, \hat{\boldsymbol{h}}_{m}\right):=\log \left(1+\frac{\left|\left\langle\hat{\boldsymbol{h}}_{m}, \boldsymbol{w}_{\pi(m)}\right\rangle\right|^{2}}{\sigma^{2}|\mathcal{S}| / P+\sum_{l \in \mathcal{S} \backslash\{m\}}\left|\left\langle\hat{\boldsymbol{h}}_{m}, \boldsymbol{w}_{\pi(l)}\right\rangle\right|^{2}}\right)
$$

Throughout the paper we assume maximum sum rate scheduling, for instance, with perfect CSIT the optimal user set $\mathcal{S}_{H}$ and mapping $\pi_{H}$ is given as

$$
\left(\mathcal{S}_{H}, \pi_{H}\right)=\underset{\substack{\mathcal{S} \subseteq \mathcal{U} \\ \pi: \mathcal{S} \rightarrow[\mathcal{C}]}}{\arg \max } R(\pi, \mathcal{S}, H) .
$$

However, due to the rate-constrained feedback channel, the base station takes its decisions based solely on partial CSIT. Partial CSIT message of each user $m \in \mathcal{U}$ contains channel direction information (CDI) $\boldsymbol{\nu}_{m} \in \mathcal{V}$ which is an element of the feedback codebook $\mathcal{V} \subset \mathbb{S}^{n_{t}-1}$ of size $|\mathcal{V}|=2^{B}$ and channel quality information (CQI) given by a scalar $\vartheta_{m} \in \mathbb{R}$. The feedback codebook is a priori known to all users and the base station. Moreover, CQI is perfectly transferred to the base station, which is a typical assumption, see e.g. [5].

If the beamforming vectors are restricted to a fixed codebook $\mathcal{C}$ the scheduling decision based on partial CSIT $V=$ $\left\{\vartheta_{m} \cdot \boldsymbol{\nu}_{m}\right\}_{m \in \mathcal{U}}$ of all users $m \in \mathcal{U}$ can be found by solving

$$
\left(\mathcal{S}_{V}, \pi_{V}\right)=\underset{\substack{\mathcal{S} \subseteq \mathcal{U} \\ \pi: \mathcal{S} \rightarrow[\mathcal{C}]}}{\arg \max } R(\pi, \mathcal{S}, V),
$$

\footnotetext{
${ }^{1}$ This condition means that the codebook constitutes a tight frame for $\mathbb{C}^{n_{t}}$ with frame constant $A$
} 
where $R(\pi, \mathcal{S}, V)=\sum_{m \in \mathcal{S}} r_{m}\left(\pi, \mathcal{S}, \vartheta_{m} \boldsymbol{\nu}_{m}\right)$. Equation (3) is a combinatorial problem that can be solved either by a brute force search over the user sets $\mathcal{S} \subseteq \mathcal{U}$, with $|\mathcal{S}| \leq n_{s}$, and the mappings $\pi: \mathcal{S} \rightarrow[\mathcal{C}]$ or more efficiently in a greedy fashion [13], [7]. Clearly, the decisions in (3) should match with the optimal decision (2) as good as possible. This is the motivation for the following RA scheme.

\section{RATE APPROXIMATION}

\section{A. RA Key Inequality}

The key idea of the RA scheme is to minimize the worst case rate mismatch between the individual user rates in (2) and (3) a priori and independent of the (unknown) scheduling decision. The feedback message is selected to make this error as small as possible.

Consider any baseline transmit scheme with perfect CSIT and sum rate $R(H)$. Define the average rate gap between the baseline transmit scheme and beamforming based on a fixed codebook with perfect CSIT as

$$
\Delta R_{\mathrm{CSIT}}:=\mathbb{E}_{H}\left[R(H)-R\left(\pi_{H}, \mathcal{S}_{H}, H\right)\right]
$$

and the average rate gap between the real sum rates $R(\pi, \mathcal{S}, H)$ and the approximated (based on partial CSIT) sum rates $R(\pi, \mathcal{S}, V)$ for a given user set $\mathcal{S}$ and mapping $\pi$ as:

$$
\Delta R(\pi, \mathcal{S}):=\mathbb{E}_{H}[R(\pi, \mathcal{S}, H)-R(\pi, \mathcal{S}, V)]
$$

Now, the rate gap between the baseline transmit scheme with perfect CSIT and beamforming based on a fixed codebook with partial CSIT can be bounded from above by

$$
\begin{aligned}
\Delta R & =\mathbb{E}_{H}\left[R(H)-R\left(\pi_{V}, \mathcal{S}_{V}, H\right)\right] \\
& =\Delta R_{\mathrm{CSIT}}+\mathbb{E}_{H}\left[R\left(\pi_{H}, \mathcal{S}_{H}, H\right)-R\left(\pi_{V}, \mathcal{S}_{V}, H\right)\right] \\
& =\Delta R_{\mathrm{CSIT}}+\Delta R\left(\pi_{H}, \mathcal{S}_{H}\right)+\mathbb{E}_{H}\left[R\left(\pi_{H}, \mathcal{S}_{H}, V\right)-R\left(\pi_{V}, \mathcal{S}_{V}, H\right)\right] \\
& \leq \Delta R_{\mathrm{CSIT}}+\Delta R\left(\pi_{H}, \mathcal{S}_{H}\right)-\Delta R\left(\pi_{V}, \mathcal{S}_{V}\right) \\
& \leq \Delta R_{\mathrm{CSIT}}+2 \cdot \mathbb{E}_{H}\left[\sum_{m \in \mathcal{S}_{H} \cup \mathcal{S}_{V}} \max _{\substack{\left.\pi_{\mathcal{S}} \mathcal{S}_{m} \rightarrow \mathcal{C}\right]\\
}} \mid r_{m}\left(\pi, \mathcal{S}, \hat{\boldsymbol{h}}_{m}\right)-r_{m}\left(\pi, \mathcal{S}, \vartheta_{m} \boldsymbol{\nu}_{m}\right)\right],
\end{aligned}
$$

where (4) must hold since $\pi_{V}$ is the optimal mapping of users to beamforming vectors under the channel state information $V$. In (5) we defined the set of user selections with maximal cardinality $n_{s}$

$$
\mathcal{S}_{m}:=\left\{\mathcal{S} \subseteq \mathcal{U} \mid m \in \mathcal{S} \text { and }|\mathcal{S}| \leq n_{s}\right\}
$$

which include user $m$. Moreover, we exploited that the rate gap $\Delta R\left(\pi_{H}\right)-\Delta R\left(\pi_{V}\right)$ is bounded from above by the worst case rate gap

$$
\Delta R_{\mathrm{RA}}:=2 \cdot \mathbb{E}_{H}\left[\sum_{m \in \mathcal{S}_{H} \cup \mathcal{S}_{V}} \max _{\substack{\mathcal{S} \in \mathcal{S} \rightarrow[\mathcal{S}] \\ \pi: \mathcal{S} \rightarrow}}\left|r_{m}\left(\pi, \mathcal{S}, \hat{\boldsymbol{h}}_{m}\right)-r_{m}\left(\pi, \mathcal{S}, \vartheta_{m} \boldsymbol{\nu}_{m}\right)\right|\right]
$$

From (5) we observe the following strategy which is the motivation for the RA scheme, described in the next subsection.

Observation 1. To control $\Delta R_{\mathrm{RA}}$ each user needs to individually minimize the individual rate gap $\left|r_{m}\left(\pi, \mathcal{S}, \hat{\boldsymbol{h}}_{m}\right)-r_{m}\left(\pi, \mathcal{S}, \vartheta_{m} \boldsymbol{\nu}_{m}\right)\right|$ for any $\mathcal{S} \in \mathcal{S}_{m}$ and mapping $\pi: \mathcal{S} \rightarrow[\mathcal{C}]$.

\section{B. RA Feedback Scheme}

To determine its feedback message each user $m \in \mathcal{U}$ must find a tuple $\left(\vartheta_{m}, \boldsymbol{\nu}_{m}\right) \in(\mathbb{R}, \mathcal{V})$ that minimizes the RA distance 2

$$
d(\boldsymbol{x}, \boldsymbol{y})=\max _{\substack{S \in \mathcal{S}_{m} \\ \pi: \mathcal{S} \rightarrow[\mathcal{C}]}}\left|r_{m}(\pi, \mathcal{S}, \boldsymbol{x})-r_{m}(\pi, \mathcal{S}, \boldsymbol{y})\right|
$$

Hence, each user $m \in \mathcal{U}$ finds its feedback message by solving

$$
\left(\vartheta_{m}, \boldsymbol{\nu}_{m}\right)=\underset{\substack{\vartheta \in \mathbb{R} \\ \boldsymbol{\nu} \in \mathcal{V}}}{\arg \min } d\left(\hat{\boldsymbol{h}}_{m}, \vartheta \boldsymbol{\nu}\right)
$$

The RA scheme can be easily extended to users with multiple receive antennas $n_{r}>1$. In this case for each scheduling decision $\pi: \mathcal{S} \rightarrow[\mathcal{C}]$ the optimal receive filter can be considered in the RA distance according to

$$
r_{m}\left(\pi, \mathcal{S}, \lambda_{m} \boldsymbol{h}_{m}\right)=\max _{\boldsymbol{u} \in \mathbb{C}^{n_{r}}} r_{m}\left(\pi, \mathcal{S}, \boldsymbol{H}_{m}^{H} \boldsymbol{u}\right) .
$$

\footnotetext{
${ }^{2} \mathrm{~A}$ closer look reveals that it is neither in all cases a distance on $\mathbb{C}^{n_{t}}$ nor on the Grassmann manifold.
} 


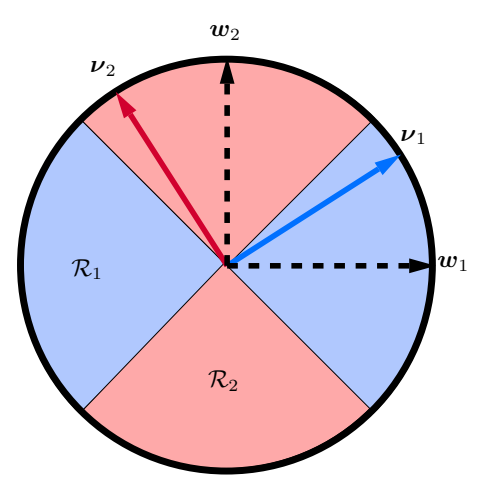

a)

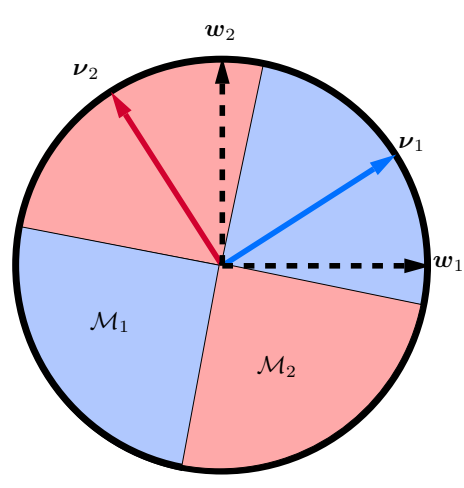

b)

Fig. 1. Toy example in $\mathbb{R}^{2}$, with $n_{s}=2, n_{t}=2$ and $n_{r}=1$, hence, $\boldsymbol{h}_{m} \in \mathbb{R}^{2}$. The transmit codebook (dashed black arrows) is given by the columns of the identity matrix and the feedback codebook (solid blues and red arrows) is given by a rotated version of the transmit codebook. The CQI is equal to the receive SNR $\vartheta_{m}^{2}=\lambda_{m}^{2}$. Comparing the feedback decisions taken under the RA distance a) and minimum chordal distance b). The sets $\mathcal{R}_{i}$ and $\mathcal{M}_{i}$ show the channel directions that result in feeding back $\nu^{[i]}$ under the RA distance and the choral distance, respectively.

Although not apparent at this point let us indicate some relevant properties of the RA scheme: first, in the RA distance $d(\cdot, \cdot)$ the transmit codebook matters which seems good engineering practice as we use all the available information. Second, the terminals provide an uniform error which indicates how well the rates are approximated and leads to inherent robustness. This becomes particularly beneficial in the LTE multi antenna case where channel state information is averaged over the subcarriers (see Simulations in Section V). Third, the RA scheme is amendable to codebook optimization based on the RA distance function (7); in [14] we presented a codebook optimization algorithm for the RA scheme which is based on the Lloyd algorithm.

Finally, consider the example in Figure 1 which establishes that the RA distance indeed yields different feedback decisions compared to the standard chordal distance [5]

$$
d_{C}\left(\boldsymbol{h}_{m}, \boldsymbol{\nu}\right)=\sqrt{1-\left|\left\langle\boldsymbol{h}_{m}, \boldsymbol{\nu}\right\rangle\right|^{2}}
$$

The RA scheme's feedback decisions is obviously more oriented towards the transmit codebook. In the following we analyze its performance. Moreover, as a by-product a simpler distance is derived which is easier to calculate than the computationally complex RA distance.

\section{Performance Analysis}

\section{A. Benchmarking Strategy}

Many papers prove that a particular transmission scheme achieves the optimal multiuser multiplexing gain. That is, for sufficiently large $|\mathcal{U}|$ the sum rate scales like $n_{t} \log \log |\mathcal{U}|$. For instance this was shown for random beamforming [15], ZF [16], UB [17] and RA [18]. However, since rates and the number of users are finite in a practical system, the significance of these asymptotic results can at least be questioned. Putting it the other way around: two methods achieving the optimal gain might behave completely different in a practical system.

Our analysis is different and more inspired by the finite user results in [5] and [19]. We assume: the number of users is equal to the number of transmit antennas $|\mathcal{U}|=n_{t}$, all users are active $\mathcal{S}=\mathcal{U}$ and the transmit codebook $\mathcal{C}$ constitutes an ONB which corresponds to UB. This assumptions enable stringent comparison to Jindal's result in [5] with ZF beamforming. Later, in Section IV-C we consider also user selection $\mathcal{S} \subseteq \mathcal{U}$ and general codebooks.

In the remainder of this section we will solely evaluate $\Delta R_{\mathrm{RA}}$ in [6]. The term $\Delta R_{\mathrm{CSIT}}$ in [5] was analyzed in [20] and [19], where it is shown that for a certain SNR range (in the low SNR regime) $\Delta R_{\text {CSIT }}$ can be even negative.

\section{B. Uniform RA Error with $U B$}

For the ease of presentation define the normalized effective channel $\boldsymbol{h}_{m}=\hat{\boldsymbol{h}}_{m} /\left\|\hat{\boldsymbol{h}}_{m}\right\|_{2}$ and the receive SNR (normalized to the number $n_{t}$ of transmit antennas) of user $m \in \mathcal{U}$ as

$$
\lambda_{m}^{2}:=\frac{P\left\|\hat{\boldsymbol{h}}_{m}\right\|_{2}^{2}}{n_{t} \sigma^{2}} .
$$

Let us first provide a general expression for the maximum in (6) which gives us a hint how the RA scheme operates. Note that when the RA scheme operates on a unitary transmit codebook we will denote this scheme by RA-UB. 


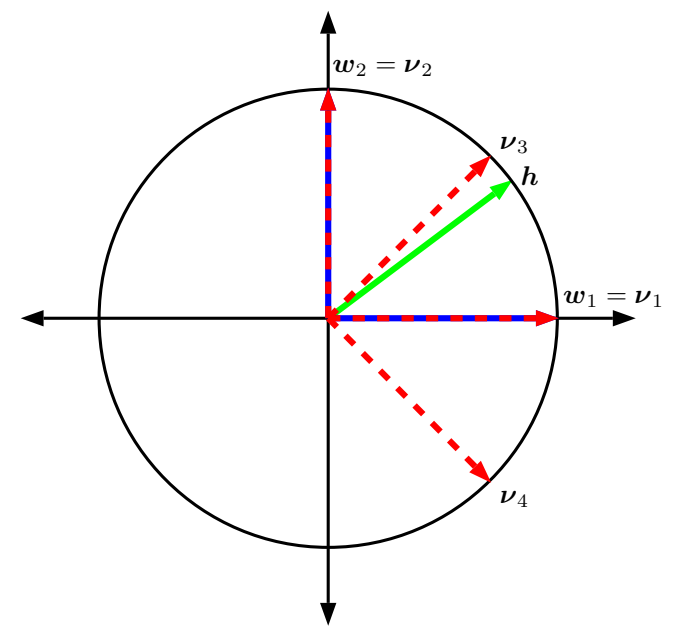

Fig. 2. Example in $\mathbb{R}^{2}$, with $n_{t}=2$ and $n_{r}=1$. The suboptimal feedback strategy considered in Lemma 1 selects $\boldsymbol{\nu}_{1}$ since $\left|\left\langle\boldsymbol{\nu}_{1}, \boldsymbol{w}^{*}\right\rangle\right| \geq\left|\left\langle\boldsymbol{h}, \boldsymbol{w}^{*}\right\rangle\right|$ must hold and in this example $\boldsymbol{w}^{*}=\boldsymbol{w}_{1}$. By contrast, the minimum chordal distance 9 selects $\boldsymbol{\nu}_{3}$ since $d_{C}(\boldsymbol{h}, \boldsymbol{\nu})$ is minimized by $\boldsymbol{\nu}=\boldsymbol{\nu}_{3}$.

Lemma 1. If $\mathcal{U}=\mathcal{S}=\left\{1,2, \ldots, n_{t}\right\}$ and $\mathcal{C} \subseteq \mathcal{V}$ then for some pair $\left(\boldsymbol{h}_{m}, \boldsymbol{\nu}\right)$ under the $R A-U B$ scheme equation 100 holds,

$$
d\left(\hat{\boldsymbol{h}}_{m}, \vartheta \boldsymbol{\nu}\right) \leq \max _{\boldsymbol{w} \neq \boldsymbol{w}^{*}} \log \left(1+\frac{\lambda_{m}^{2}\left(\left.||\left\langle\boldsymbol{h}_{m}, \boldsymbol{w}\right\rangle\right|^{2}-|\langle\boldsymbol{\nu}, \boldsymbol{w}\rangle|^{2}\left|+\frac{|\langle\boldsymbol{\nu}, \boldsymbol{w}\rangle|^{2}}{\left\langle\left.\left\langle\boldsymbol{\nu}, \boldsymbol{w}^{*}\right\rangle\right|^{2}\right.}\right|\left|\left\langle\boldsymbol{\nu}, \boldsymbol{w}^{*}\right\rangle\right|^{2}-\left|\left\langle\boldsymbol{h}_{m}, \boldsymbol{w}^{*}\right\rangle\right|^{2} \mid\right)}{1+\lambda_{m}^{2}\left(1-\max \left\{\left|\left\langle\boldsymbol{h}_{m}, \boldsymbol{w}\right\rangle\right|^{2},|\langle\boldsymbol{\nu}, \boldsymbol{w}\rangle|^{2}\right\}\right)}\right)
$$

where we defined $\boldsymbol{w}^{*}$ by $\left|\left\langle\boldsymbol{h}_{m}, \boldsymbol{w}\right\rangle\right|^{2} \leq\left|\left\langle\boldsymbol{h}_{m}, \boldsymbol{w}^{*}\right\rangle\right|^{2}$ for all $\boldsymbol{w} \in \mathcal{C}$. The strategy minimizing the upper bound is to pick $\boldsymbol{\nu}$ close to $\boldsymbol{h}_{m}$ (in the chordal distance) constrained by $\left|\left\langle\boldsymbol{\nu}, \boldsymbol{w}^{*}\right\rangle\right|^{2} \geq\left|\left\langle\boldsymbol{h}_{m}, \boldsymbol{w}^{*}\right\rangle\right|^{2}$.

The proof can be found in Appendix $\mathrm{A}$

Observation 2. The suboptimal strategy in Lemma 1 supports the intuition that the error for the best beamformer should be set to zero by the applied feedback strategy, see the example in Figure 2

So far we are not able to effectively bound $\Delta R_{\mathrm{RA}}$ which is now settled based on Lemma 1 . The following lemma shows that $\Delta R_{\mathrm{RA}}$ remains bounded when the SNR increases and that the rate error depends solely on the function

$$
D_{m}(B):=\min _{\mathcal{V},|\mathcal{V}|=2^{B}} \mathbb{E}_{H}\left[\left.\frac{1}{1-\tilde{\lambda}_{m}} \cdot \min _{\substack{1>\tilde{\vartheta}_{m}>0 \\ \boldsymbol{\nu} \in \mathcal{V}}} \max _{\boldsymbol{w} \in \mathcal{C}}\left|\tilde{\lambda}_{m}\right|\left\langle\boldsymbol{h}_{m}, \boldsymbol{w}\right\rangle\right|^{2}-\tilde{\vartheta}_{m}|\langle\boldsymbol{\nu}, \boldsymbol{w}\rangle|^{2} \mid\right],
$$

where we defined $\tilde{\lambda}_{m}=\frac{\lambda_{m}^{2}}{1+\lambda_{m}^{2}}$ and $\tilde{\vartheta}_{m}=\frac{\vartheta_{m}^{2}}{1+\vartheta_{m}^{2}}$ in the proof of Lemma 1 and $B$ is the number of feedback bits.

Lemma 2. If $\mathcal{U}=\mathcal{S}=\left\{1,2, \ldots, n_{t}\right\}$ and $\mathcal{C} \subseteq \mathcal{V}$ then under the $R A-U B$ scheme

$$
\Delta R_{R A} \leq 2 \sum_{m=1}^{n_{t}} \log \left(1+\min _{\epsilon>0} \frac{(1+\epsilon) D_{m}(B)}{1+\frac{\epsilon}{n_{t}-1} D_{m}(B)}\right)
$$

The proof can be found in Appendix $\mathbb{B}$. The following lemma gives a fundamental bound on $D_{m}(B)$.

Lemma 3. If the transmit codebook $\mathcal{C}$ is unitary, then

$$
D_{m}(B) \leq c\left(n_{t}\right) \mathbb{E}_{H}\left[\lambda_{m}^{2}\right] 2^{-\frac{B}{n_{t}-1}},
$$

with

$$
c\left(n_{t}\right)=\left(\Theta\left(\mathcal{B}_{2}^{n_{t}-1}\right)\left(\begin{array}{c}
2 n_{t}-2 \\
n_{t}-1
\end{array}\right) \frac{\Gamma\left(1+\frac{n_{t}-1}{2}\right) \sqrt{n_{t}}}{\left(n_{t}-1\right) ! \pi^{\frac{n_{t}-1}{2}}}\right)^{\frac{1}{n_{t}-1}}
$$

and $B \geq \frac{\left(n_{t}-1\right)}{2} \log \left[\left(n_{t}-1\right) \sqrt{n_{t}-1}\right]$. For $n_{t}-1$ small tight bounds are known for the covering density $\Theta\left(\mathcal{B}_{2}^{n_{t}-1}\right)$, e.g. $\Theta\left(\mathcal{B}_{2}^{2}\right) \leq 1.2091$ (Kershner, 1939), $\Theta\left(\mathcal{B}_{2}^{3}\right) \leq 1.4635$ (Bambah, 1954), $\Theta\left(\mathcal{B}_{2}^{4}\right) \leq 1.7655$ (Delone \& Ryshkov, 1963). For $n_{t}-1 \geq 3$ the Rogers bound [21] $\Theta\left(\mathcal{B}_{2}^{n_{t}-1}\right)<4\left(n_{t}-1\right) \log \left(n_{t}-1\right)$ can be used.

The complete proof can be found in the Appendix $\mathrm{C}$ Note that $c\left(n_{t}\right)$ is close to unity and falls below unity not before $n_{t} \geq 14$, as required for improved scaling compared to Jindal's result. As the following illustration for the case $n_{t}=3$ shows, this is simply an artefact of the proof technique. 


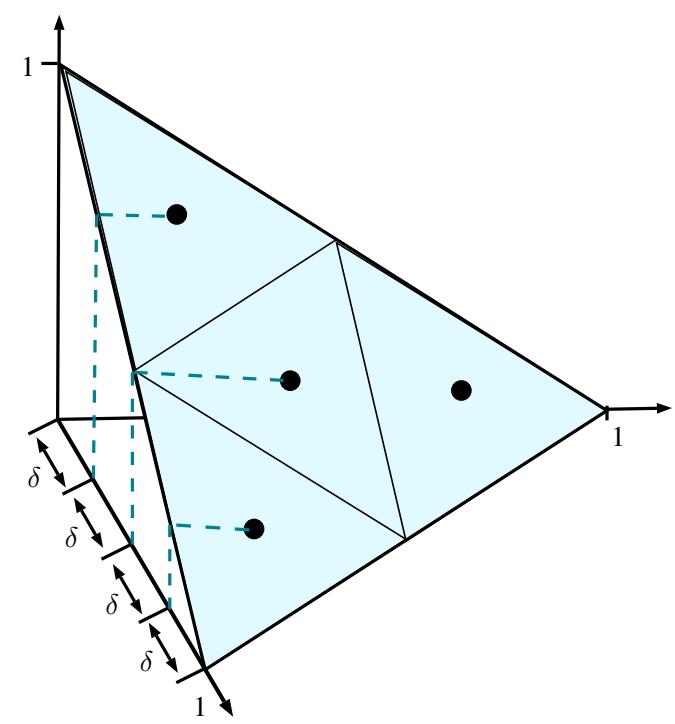

Fig. 3. The standard 2-simplex in 3 dimensions. The projection of the quantization points $\mathcal{Q}$ on the coordinate axes implies a worst case quantization error $\delta$.

Without loss of generality, we assume the unitary transmit codebook is given by the standard ONB. We drop the user index $m$ and define from its channel direction $\boldsymbol{h}$ the real positive vectors $\boldsymbol{\psi}=\left(\psi_{1}, \ldots, \psi_{3}\right)$ with $\psi_{n}:=\left|\left\langle\boldsymbol{h}, \boldsymbol{w}_{\pi(n)}\right\rangle\right|^{2}$ and $\phi^{\nu}=\left(\phi_{1}^{\nu}, \ldots, \phi_{3}^{\nu}\right)$ with $\phi_{n}^{\nu}:=\left|\left\langle\boldsymbol{\nu}, \boldsymbol{w}_{\pi(n)}\right\rangle\right|^{2}$ for each $\boldsymbol{\nu} \in \mathcal{V}$. Per definition, all these vectors have unit $\ell_{1}$-norm, $\|\boldsymbol{\psi}\|_{1}=\left\|\boldsymbol{\phi}^{\boldsymbol{\nu}}\right\|_{1}=1$ and, hence, define points on the standard 2-simplex. Further, $\max _{\pi}\left|\psi_{n}-\phi_{n}^{\nu}\right|=\left\|\boldsymbol{\psi}-\boldsymbol{\phi}^{\boldsymbol{\nu}}\right\|_{\infty}$ defines a distance between two points on the standard 2-simplex. Hence, for a given feedback codebook $\mathcal{V}$ we can define the Voronoi region around the point $\phi^{\nu}$ for a particular $\boldsymbol{\nu} \in \mathcal{V}$ as $V\left(\phi^{\nu}\right)=\left\{\boldsymbol{x} \in \mathbb{R}_{+}^{3}:\left\|\boldsymbol{x}-\boldsymbol{\phi}^{\nu}\right\|_{\infty}<\left\|\boldsymbol{x}-\phi^{\boldsymbol{\xi}}\right\|_{\infty}, \forall \boldsymbol{\xi} \in \mathcal{V}, \boldsymbol{\xi} \neq \boldsymbol{\nu}\right\}$. If $B \in\{1,2,4, \ldots\}$ and $n_{t}=3$, the feedback codebook can be chosen such that the Voronoi regions are 2-simplices with edge length $\tilde{\delta} \leq \sqrt{2}$. Now, using the symmetry of the covering and projecting the quantization points back on the coordinate axes (see Figure 3) we get $\max _{\boldsymbol{x} \in V\left(\phi^{\nu}\right)}\left\|\boldsymbol{x}-\boldsymbol{\phi}^{\boldsymbol{\nu}}\right\|_{\infty}=\tilde{\delta} / \sqrt{8}=\delta$.

Now we can compute the volumes of the 2 -simplices (the standard simplex and the scaled simplex) and proceed as in the proof of Lemma 3 to obtain the result

$$
\delta=\max _{\boldsymbol{x} \in V\left(\phi^{\nu}\right)}\left\|\boldsymbol{x}-\boldsymbol{\phi}^{\nu}\right\|_{\infty}=2^{-\frac{B}{n_{t}-1}-1} .
$$

Hence, if $n_{t}=3$ and Rayleigh fading is assumed (i.e. $\hat{\boldsymbol{h}}_{m, i} \sim \mathcal{C N}(0,1)$ for $i=1, \ldots, n_{t}$ and $m \in \mathcal{U}$ ), the rate loss due to the rate-constrained feedback channel scales like

$$
\Delta R_{\mathrm{RA}} \leq 2 \sum_{m=1}^{n_{t}} \log \left(1+\min _{\epsilon>0} \frac{(1+\epsilon) \mathbb{E}_{H}\left[\frac{\tilde{\lambda}_{m}}{1-\lambda_{m}}\right] 2^{-\frac{B}{n_{t}-1}-1}}{1+\mathbb{E}_{H}\left[\frac{\tilde{\lambda}_{m}}{1-\lambda_{m}}\right] \frac{\epsilon}{n_{t}-1} 2^{-\frac{B}{n_{t}-1}-1}}\right) \leq 2 \sum_{m=1}^{n_{t}} \log \left(1+\frac{P}{\sigma^{2}} 2^{-\frac{B}{n_{t}-1}-1}\right) .
$$

Therefore, we have an improvement of $n_{t}-1$ bits in the exponential term compared to Jindal's result for ZF with feedback based on minimizing the chordal distance (see [5]), under the very same assumptions.

\section{Uniform RA Error with User Selection and General Codebooks}

In this subsection we no longer assume unitary transmit codebooks and allow user selection at the base station.

Theorem 1. Assuming Rayleigh fading, arbitrary transmit codebooks and user selection $\mathcal{S} \subseteq \mathcal{U}=\left\{1,2, \ldots, n_{t}\right\}$, then for any $\mathcal{V}$ under the $R A$ scheme

$$
\Delta R_{R A} \leq 4 n_{s} \log \left(1+\frac{P n_{t}}{\sigma^{2}} \mathbb{E}_{H}\left[\left.\min _{\boldsymbol{\nu} \in \mathcal{V}} \max _{\boldsymbol{w} \in \mathcal{C}}||\langle\boldsymbol{h}, \boldsymbol{w}\rangle\right|^{2}-|\langle\boldsymbol{\nu}, \boldsymbol{w}\rangle|^{2} \mid\right]\right) .
$$

The proof can be found in Appendix $\mathrm{D}$, The expected value

$$
\hat{D}_{m}(B):=\min _{\mathcal{V},|\mathcal{V}|=2^{B}} \mathbb{E}_{H}\left[\left.\min _{\boldsymbol{\nu} \in \mathcal{V}} \max _{\boldsymbol{w} \in \mathcal{C}}||\langle\boldsymbol{h}, \boldsymbol{w}\rangle\right|^{2}-|\langle\boldsymbol{\nu}, \boldsymbol{w}\rangle|^{2} \mid\right]
$$

has been shown to be analytically tractable, in the previous section, for unitary transmit codebooks. For codebooks constituting a tight frame (see the condition (1) we devise the following corollary. 


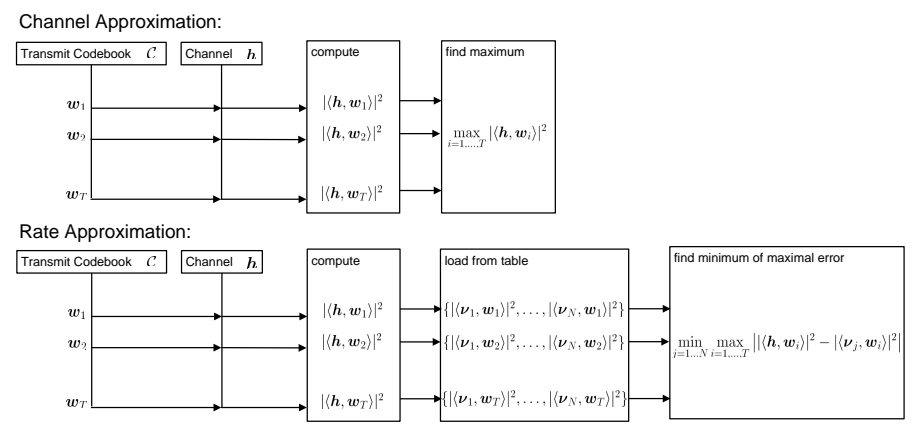

Fig. 4. Schematic comparison of the CDI computation at the user side; for the efficient RA distance (bottom) and chordal distance (top).

Corollary 1. If the transmit codebook $\mathcal{C}$ is a tight frame, then $\hat{D}_{m}(B) \leq A \cdot c\left(n_{t}\right) 2^{-\frac{B}{n_{t}-1}}$, where $c\left(n_{t}\right)$ is defined in (11) and $A$ is the frame constant in (1).

The proof is a simple extension of Lemma 3 and omitted. The previous result is remarkable since all the $2^{n_{t}}$ possible user rates are uniformly recovered at the base station with better scaling properties than the classical result. The RA scheme is now applied in a practical scenario.

\section{Practical Considerations and Simulations}

\section{A. Efficient and Robust Feedback Protocol}

Mobile user equipments usually have limited computing capabilities, therefore, most systems require that the complexity at the user side is as low as possible. Hence, solving the full rate approximation problem (the min-max problem (8)) may not be feasible. Fortunately, our analysis in Section IV yields the (suboptimal) distance function

$$
d_{S}(\boldsymbol{h}, \boldsymbol{\nu})=\left.\max _{\boldsymbol{w} \in \mathcal{C}}||\langle\boldsymbol{h}, \boldsymbol{w}\rangle\right|^{2}-|\langle\boldsymbol{\nu}, \boldsymbol{w}\rangle|^{2} \mid,
$$

which can be used at the user side to uniformly bound the rate approximation error $\Delta R_{\mathrm{RA}}$ (6). Further, we define the CQI reported by user $m$ as

$$
\vartheta_{m}^{2}=\lambda_{m}^{2}\left|\left\langle\boldsymbol{h}_{m}, \boldsymbol{\nu}_{m}\right\rangle\right|^{2}
$$

which can be interpreted as the effective channel gain of user $m$ over the quantized channel $\nu_{m}$. Equation (13) captures two important aspects. On the one hand, if the CDI is equal to the channel direction, the user gets no penalty $\left(\left|\left\langle\boldsymbol{h}_{m}, \boldsymbol{\nu}_{m}\right\rangle\right|^{2}=1\right)$ on the other hand if the CDI is orthogonal to the channel direction, the effective channel is zero $\left(\left|\left\langle\boldsymbol{h}_{m}, \boldsymbol{\nu}_{m}\right\rangle\right|^{2}=0\right)$. Hence, the CQI (13) reflects the receive SNR and the quantization error, which is also in accordance with the results in [16]. In Algorithm 1 the efficient feedback protocol is summarized.

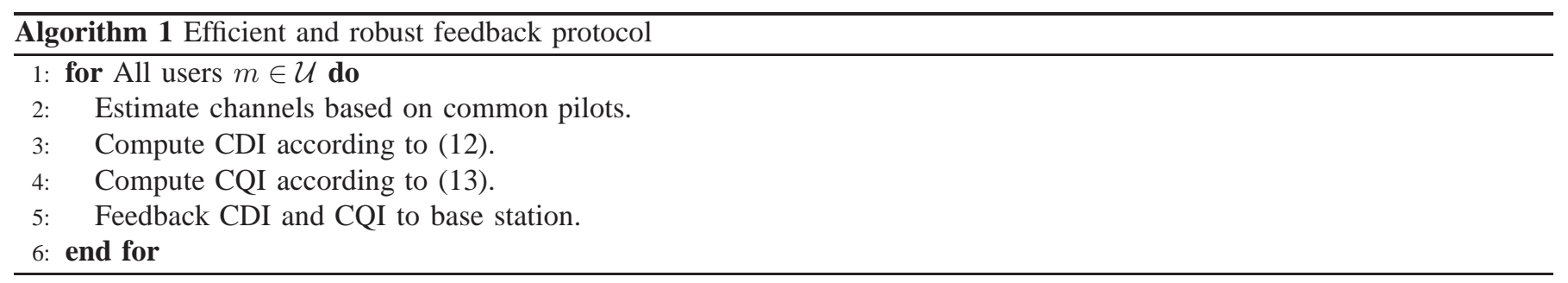

Complexity of the proposed feedback protocol: Figure 4 (bottom) shows a flow chart of the CDI computation with the proposed feedback protocol using the distance function $d_{S}(\boldsymbol{h}, \boldsymbol{\nu})$ defined in (12). We point out that the terms $\left|\left\langle\boldsymbol{\nu}_{j}, \boldsymbol{w}_{i}\right\rangle\right|$, for $i=1, \ldots,|\mathcal{C}|$ and $j=1, \ldots,|\mathcal{V}|$, only need to be computed ones and can be stored in the memory. Therefore, during the feedback phase user $m$ must only compute $\left|\left\langle\boldsymbol{h}_{m}, \boldsymbol{w}_{i}\right\rangle\right|^{2}$ for all $i=1, \ldots,|\mathcal{C}|$ and the difference $\left.||\left\langle\boldsymbol{h}_{m}, \boldsymbol{w}_{i}\right\rangle\right|^{2}-\left|\left\langle\boldsymbol{\nu}_{j}, \boldsymbol{w}_{i}\right\rangle\right|^{2} \mid$ for all $i, j$. Figure 4 (top) shows the steps that need to be performed to compute the CDI based on the chordal distance (9). To compute the chordal distance each user must compute $\left|\left\langle\boldsymbol{h}_{m}, \boldsymbol{\nu}_{i}\right\rangle\right|$ for all $i=1, \ldots,|\mathcal{V}|$.

If we assume a fixed transmit codebook $\mathcal{C}$ and a feedback codebook $\mathcal{V}$ with $2^{B}$ elements, the complexity of computing the CDI based on (9) or (12) is asymptotically equal, i.e., using Landau notation $O\left(2^{B}\right)$. However, this result is only valid for $2^{B}$ growing asymptotically large. For small values $B=1,2,3,4,8$ and $|\mathcal{C}|=8$ the number of scalar products that needs to be evaluated are summarized in Table [. 
TABLE I

Number of SCALAR Products that NeEd to be EVAluated to solve MD 9 AND the Proposed RA distance 12

\begin{tabular}{|l|l|l|}
\hline B & MD 90 & proposed RA (12) \\
\hline 1 & 2 & 16 \\
2 & 4 & 32 \\
3 & 8 & 64 \\
4 & 16 & 128 \\
8 & 256 & 2048 \\
\hline
\end{tabular}

\section{B. Simulations}

In the simulations we consider a LTE like system architecture. That is, multiple base stations transmit to multiple users using the spectrum. The spectrum is divided in orthogonal subcarriers using orthogonal frequency-division multiplexing OFDM. In the sequel we use a frequency reuse factor of one, i.e., each base station uses the whole frequency band. Since, we assume no cooperation between the base stations inter cell interference is indispensable. In the sequel the channel from base station $b$ to user $m$ on subcarrier $f$ is given by $\boldsymbol{H}_{m, b}(f) \in \mathbb{C}^{n_{r} \times n_{t}}$.

The transmit protocol can be summarized as follows. First, each base station transmits orthogonal common pilots. Then, each user quantizes and feeds back its channel state information. Based on the quantized channel state information each base station solves the scheduling problem (3). Finally, dedicated (i.e. precoded) pilots are transmitted by all base stations.

TABLE II

Simulation PARAMETERS

\begin{tabular}{|l|l|}
\hline Parameter & Value/Assumption \\
\hline Number of base stations & 3 \\
Frequency reuse & full \\
Number of users $|\mathcal{U}|$ & 30 (uniformly distributed) \\
Number of transmit antennas $n_{t}$ & 4 (uncorrelated) \\
Number of receive antennas $n_{r}$ & 1 or 2 (uncorrelated) \\
Receiver type & maximum ratio combining \\
Maximum number of scheduled users per & 4 \\
scheduling block $n_{s}$ & \\
Equivalent SNR & $153 \mathrm{~dB}$ \\
LTE carrier frequency / bandwidth & $2 \mathrm{GHz} / 10 \mathrm{MHz}$ \\
Number of PRB & 50 \\
Scheduling block size & $1 \mathrm{PRB}=12$ subcarrier \\
LTE channel model & SCME (urban macro) \\
Inter cell interference modeling & explicit \\
\hline
\end{tabular}

The simulation parameters are given in Table II they can be summarized as follows. 3 base stations located in 3 adjacent cells and 30 users uniformly distributed over the network area; given by a radius of 250 meter around the center of the base stations. The physical layer is configured according to LTE [22]. The base station are equipped with $n_{t}=4$ transmit antennas and each user is equipped with $n_{r}=1$ or $n_{r}=2$ receive antenna (specified in the caption). The transmit codebook and feedback codebook is given by the LTE codebook defined in [22] which has $N=16$ elements and, hence, requires $B=4$ bit to feedback back the CDI. The channels are modeled by the spatial channel model extended (SCME) [23] using the urban macro scenario.

In total 600 subcarriers per base station are available. The subcarriers are clustered in groups of $F=12$ subcarriers; one subcarrier group is denoted as physical resource block (PRB). One PRB is the smallest scheduling unit. The subcarrier indexes of PRB $p$ are collected in the index set $\mathcal{F}_{p}$. We define the average channel gain of PRB $p$ as $\sigma_{p}^{2}=1 /\left|\mathcal{F}_{p}\right| \sum_{f \in \mathcal{F}_{s}}\left\|\boldsymbol{H}_{b, m}(f)\right\|_{F}^{2}$, where $\|\boldsymbol{A}\|_{F}$ is the Frobenius norm of matrix $\boldsymbol{A}$, and assume that each user is assigned to that base station with maximal total average channel gain $1 / F \sum_{f=1}^{F} \sigma_{f}^{2}$. Each user reports one feedback message per PRB to that base station it is assigned to.

Each of the base stations runs an independent local scheduler. In every transmission interval up to $n_{s}=2$ users can be scheduled by each base station on every PRB. Scheduling is performed in a greedy fashion according to [24]. For simplicity we assume no delay in the CSIT report, scheduling, transmission or performance evaluation.

The performance is evaluated based on the network spectral efficiency which we define by $\sum_{b=1}^{B} \sum_{p=1}^{F} \sum_{m \in \mathcal{S}_{b, p}} \sum_{f \in \mathcal{F}_{p}} \log (1+$ $\left.\operatorname{SINR}_{m}(f)\right)$, where $\operatorname{SINR}_{m, b}(f)$ is the SINR of user $m$ on subcarrier $f$ and $S_{b, p}$ are the users scheduled by base station $b$ on PRB $p$.

In the simulation we compare four different feedback strategies.

1) Perfect (average) CSIT: the base station knows the channel averaged over all subcarriers perfectly, $\overline{\boldsymbol{H}}_{m}=\frac{1}{F} \sum_{f=1}^{F} \boldsymbol{H}_{m, f}$, where $\boldsymbol{H}_{m, f}$ is the channel of user $m$ on subcarrier $f$.

2) Minimum chordal distance: user $m$ determines its CDI feedback by minimizing the chordal distance (9) to the channel $\overline{\boldsymbol{h}}_{m}=\left\langle\boldsymbol{u}, \overline{\boldsymbol{H}}_{m}\right\rangle$, where $\boldsymbol{u}$ is chosen to maximize $\left|\left\langle\boldsymbol{u}, \overline{\boldsymbol{H}}_{m}\right\rangle\right|$. 


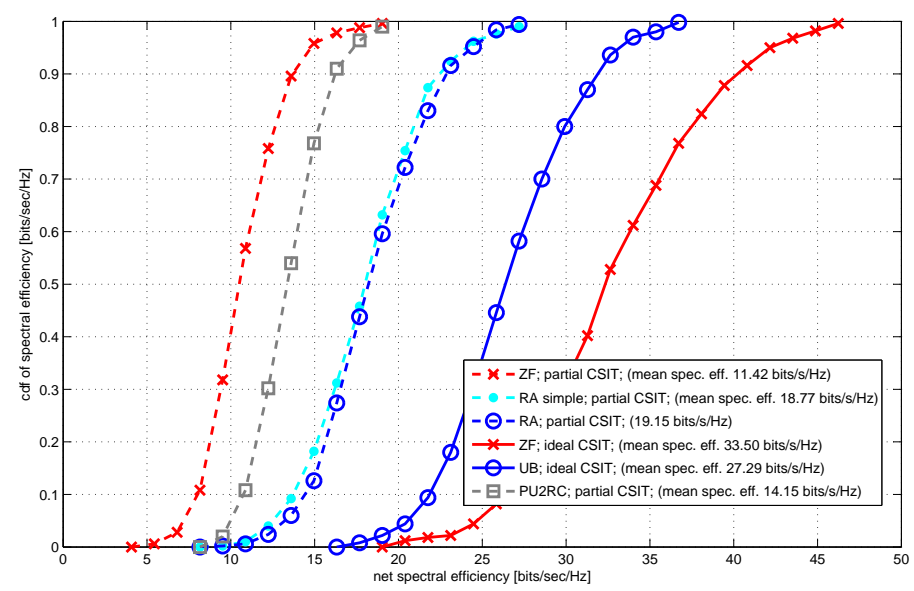

Fig. 5. System level simulation: CDF of spectral efficiency for $n_{r}=1$; Comparing PU2RC, ZF, UB and RA under ideal and partial CSIT

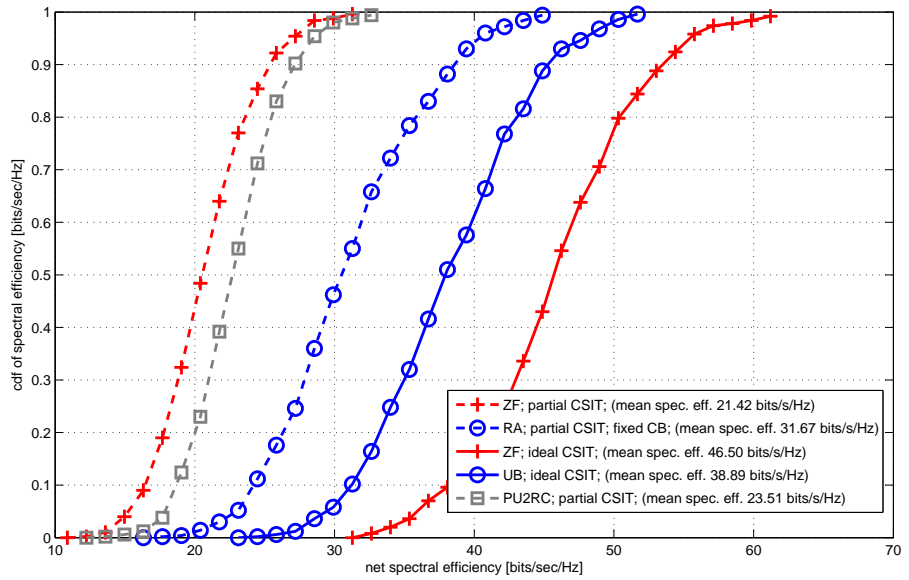

Fig. 6. System level simulation: CDF of spectral efficiency for $n_{r}=2$; Comparing PU2RC, ZF, UB and RA under ideal and partial CSIT

3) Rate Approximation as described in Section III with the rates

$$
r_{m}\left(\pi, \mathcal{S}, \lambda_{m} \boldsymbol{h}_{m}\right)=\frac{1}{F} \sum_{f=1}^{F} r_{m}\left(\pi, \mathcal{S}, \lambda_{m} \boldsymbol{h}_{m, f}\right) .
$$

4) Efficient Rate Approximation as described in Section $\mathrm{V}$-A where $\boldsymbol{h}_{m}$ is given by the average channel $\overline{\boldsymbol{h}}_{m}$ as defined for minimum chordal distance above.

Figure 5] depicts the CDF of the spectral efficiency for users with $n_{r}=1$ receive antenna. The ZF scheme is implemented according to [7]. The PU2RC scheme is based on the same transmit codebook as RA and is implemented according to [10]. We observe that with perfect CSIT ZF outperforms greedy scheduling with a fixed codebook. With partial CSIT the RA scheme significantly outperforms ZF with a gain of approximately $70 \%$. Remarkable is also the gain of about 35\% of RA over PU2RC. Moreover, Figure 5 shows that RA with the efficient distance function (12) performs very close to the full RA scheme.

Figure 6 depicts the CDF of the spectral efficiency for users with $n_{r}=2$ receive antennas. We observe that with perfect CSIT ZF outperforms greedy scheduling with a fixed codebook. With partial CSIT the RA scheme significantly outperforms all other schemes and achieves a gain of approximately $50 \%$ over ZF. Remarkable is also the 35\% gain of RA over PU2RC.

In Figure 7 we compare the performance of RA and partial CSIT ZF with different receive filters, i.e. the MMSE receive filter that maximizes the SINR of each user by considering the interference from all other users, the global ZF receive filter that tries to minimize interference from all base stations and the local ZF receive filter that considers only interference from the own base station. We observe that both transmit schemes achieve the highest network sum rate with the SINR optimal receive filter. The performance degradation with the local and global ZF receive filter are similar for both transmit schemes.

\section{CONCLUSION}

In this paper we invented and analyzed the rate approximation scheme. It was shown that each user can individually minimize its rate error a priori by selecting the feedback message in a robust fashion incorporating the transmit codebook. The respective 


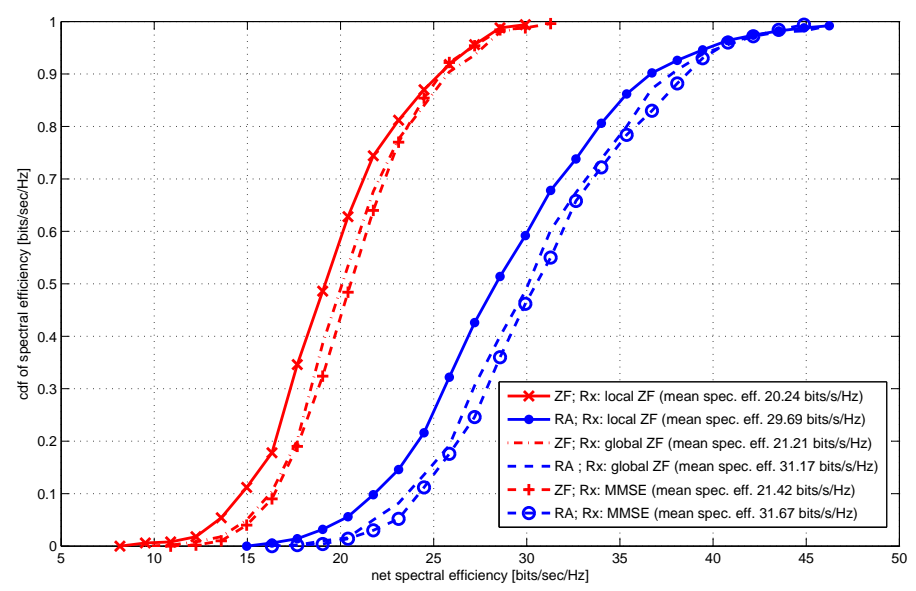

Fig. 7. System level simulation: $\mathrm{CDF}$ of spectral efficiency for $n_{r}=2$; Comparing ZF and RA with partial CSIT and different receive filters.

error expressions and feedback schemes are derived and compared to the standard expressions. It is proved that a better scaling is possible when the size of the transmit code book is small. A remarkable result is that it is often much better to reduce flexibility at the base station in favor of having more reliable CSIT.

\section{APPENDIX A}

PROOF OF LEMMA 1

Proof: Let us first drop the user index $m$, i.e. $\lambda=\lambda_{m}$ and $\boldsymbol{h}=\boldsymbol{h}_{m}$. Further abbreviate $\boldsymbol{w}=\boldsymbol{w}_{\pi(m)}, \phi=|\langle\boldsymbol{\nu}, \boldsymbol{w}\rangle|^{2}$ and $\psi=|\langle\boldsymbol{h}, \boldsymbol{w}\rangle|^{2}$ without explicitly writing the dependency of $\pi$. Using $\mathcal{U}=\mathcal{S}$ with $|\mathcal{S}|=n_{t}$ we get for any $\vartheta$ and normalized vector $\boldsymbol{\nu}$

$$
\begin{aligned}
r_{m}(\pi, \mathcal{S}, \lambda \boldsymbol{h})-r_{m}(\pi, \mathcal{S}, \vartheta \boldsymbol{\nu}) & =\log \left(\frac{\left(\lambda^{2}+1\right)\left(1+\vartheta^{2}(1-\phi)\right)}{\left(\vartheta^{2}+1\right)\left(1+\lambda^{2}(1-\psi)\right)}\right) \\
& =\log \left(\frac{1-\tilde{\vartheta} \phi}{1-\tilde{\lambda} \psi}\right)=\log \left(1+\frac{\tilde{\lambda} \psi-\tilde{\vartheta} \phi}{1-\tilde{\lambda} \psi}\right) .
\end{aligned}
$$

Here, we have set $\tilde{\vartheta}:=\frac{\vartheta^{2}}{\vartheta^{2}+1}, \quad \tilde{\lambda}:=\frac{\lambda^{2}}{\lambda^{2}+1}$. Similarly, the negative term can be rewritten as $-\log \left(\frac{1-\tilde{\vartheta} \phi}{1-\tilde{\lambda} \psi}\right)=\log \left(1+\frac{\tilde{\vartheta} \phi-\tilde{\lambda} \psi}{1-\tilde{\vartheta} \phi}\right)$. Recall, that $\phi$ and $\psi$ depend on $\boldsymbol{w}$ which in turn depends again on the mapping $\pi$. With the assumptions of this lemma we have that the set $\mathcal{S}_{m}$ of possible scheduling subsets in the definition of RA distance (7) is simple, i.e. $\mathcal{S}_{m}=\{\mathcal{U}\}$ and we get from 15 the following upper bound

$$
\begin{aligned}
d_{m}(\lambda \boldsymbol{h}, \vartheta \boldsymbol{\nu}) & =\max _{\pi}\left|r_{m}(\pi, \mathcal{S}, \lambda \boldsymbol{h})-r_{m}(\pi, \mathcal{S}, \vartheta \boldsymbol{\nu})\right| \\
& \leq \max _{\pi} \max \left[\log \left(1+\frac{\tilde{\lambda} \psi-\tilde{\vartheta} \phi}{1-\tilde{\lambda} \psi}\right), \log \left(1+\frac{\tilde{\vartheta} \phi-\tilde{\lambda} \psi}{1-\tilde{\vartheta} \phi}\right)\right] \\
& \leq \max _{\pi} \log \left(1+\max \left[\frac{\tilde{\lambda} \psi-\tilde{\vartheta} \phi}{1-\tilde{\lambda} \psi}, \frac{\tilde{\vartheta} \phi-\tilde{\lambda} \psi}{1-\tilde{\vartheta} \phi}\right]\right)
\end{aligned}
$$

which is still valid for any $\nu \in \mathcal{V}$. Now, consider the following (sub-optimal) three-step strategy for the feedback protocol: (a) define $\boldsymbol{w}^{*}:=\arg \max _{\boldsymbol{w} \in \mathcal{C}}|\langle\boldsymbol{h}, \boldsymbol{w}\rangle|^{2}$ to be the codeword nearest to true channel direction $\boldsymbol{h}$ in the chordal distance (see (9)) and (b) select then a CDI $\nu \in \mathcal{V}$ from the feedback codebook which is closer to $\boldsymbol{w}^{*}$ as $\boldsymbol{h}$ is, i.e. which has the property $\theta:=\left|\left\langle\boldsymbol{\nu}, \boldsymbol{w}^{*}\right\rangle\right|^{2} \geq\left|\left\langle\boldsymbol{h}, \boldsymbol{w}^{*}\right\rangle\right|^{2}=: \eta$. Since $\mathcal{C} \subseteq \mathcal{V}$ such vector always exists. (c) Determine the CQI $\vartheta$ by setting $\frac{\lambda^{2} \eta}{1+\lambda^{2}(1-\eta)}=\frac{\vartheta^{2} \eta}{1+\vartheta^{2}(1-\theta)}$, which yields after some calculations $\tilde{\vartheta}=\frac{\lambda^{2}}{\lambda^{2}+1} \frac{\eta}{\theta}=\tilde{\lambda} \frac{\eta}{\theta}$. The result of this strategy is that we get for the first term in the "max" of 16):

$$
\frac{\tilde{\lambda} \psi-\tilde{\vartheta} \phi}{1-\tilde{\lambda} \psi} \leq \tilde{\lambda} \cdot \frac{\psi-\frac{\eta}{\theta} \phi}{1-\tilde{\lambda} \psi} \leq \frac{\tilde{\lambda}}{1-\tilde{\lambda} \psi}\left(|\psi-\phi|+\frac{\phi}{\theta}|\theta-\eta|\right) .
$$


for any $\boldsymbol{w} \neq \boldsymbol{w}^{*}$ (which ensures that $\psi=|\langle\boldsymbol{h}, \boldsymbol{w}\rangle|^{2}<\left|\left\langle\boldsymbol{h}, \boldsymbol{w}^{*}\right\rangle\right|^{2}=\eta \leq 1$ ). Similar, for the second term in upper bound (16) we obtain

$$
\begin{aligned}
\frac{\tilde{\vartheta} \phi-\tilde{\lambda} \psi}{1-\tilde{\vartheta} \phi} & \leq \tilde{\lambda} \cdot \frac{\frac{\eta}{\theta} \phi-\psi}{1-\tilde{\lambda} \frac{\eta}{\theta} \phi} \stackrel{(\eta \leq \theta)}{\leq} \tilde{\lambda} \cdot \frac{\frac{\eta}{\theta} \phi-\psi}{1-\tilde{\lambda} \phi} \\
& \leq \frac{\tilde{\lambda}}{1-\tilde{\lambda} \phi}\left(|\psi-\phi|+\frac{\phi}{\theta}|\theta-\eta|\right)
\end{aligned}
$$

where we now need the additional property of step (b) that $\eta=\left|\left\langle\boldsymbol{h}, \boldsymbol{w}^{*}\right\rangle\right|^{2} \leq\left|\left\langle\boldsymbol{\nu}, \boldsymbol{w}^{*}\right\rangle\right|^{2}=\theta$ which proves the claim.

\section{APPENDIX B}

\section{PROOF OF LEMMA 2}

Proof: According to the rule (8), RA aims on minimizing the maximal rate error over the elements of $\mathcal{V}$. Under the assumptions of this lemma (the list of scheduling subsets is $\mathcal{S}_{m}=\{\mathcal{U}\}$ ) the maximal rate error at user $m$ achieved for given CDI $\nu_{m}$ and CQI $\vartheta_{m}$ is:

$$
d_{m}\left(\lambda_{m} \boldsymbol{h}_{m}, \vartheta \boldsymbol{\nu}\right)=\max _{\pi}\left|r_{m}\left(\pi, \mathcal{S}, \lambda_{m} \boldsymbol{h}_{m}\right)-r_{m}\left(\pi, \mathcal{S}, \vartheta_{m} \boldsymbol{\nu}_{m}\right)\right|
$$

and from (6) we have in this case:

$$
\Delta R_{\mathrm{RA}}:=2 \sum_{m \in \mathcal{S}} \mathbb{E}_{H}\left[d_{m}\left(\lambda_{m} \boldsymbol{h}_{m}, \vartheta_{m} \boldsymbol{\nu}_{m}\right)\right]
$$

Let us consider the contribution of user $m$ to this sum. Using the notation of Appendix $\mathrm{A}$ we have from (16) and Jensen's inequality that

$$
\mathbb{E}_{H}\left[d_{m}(\lambda \boldsymbol{h}, \vartheta \boldsymbol{\nu})\right] \leq \log \left(1+\mathbb{E}_{H}\left[\min _{\substack{1>\tilde{\vartheta}_{m}>0 \\ \boldsymbol{\nu} \in \mathcal{V}}} \max _{\pi} \frac{|\tilde{\lambda} \psi-\tilde{\vartheta} \phi|}{1-\tilde{\lambda} \psi}\right]\right)+\log \left(1+\mathbb{E}_{H}\left[\min _{\substack{1>\tilde{\vartheta}_{m}>0 \\ \boldsymbol{\nu} \in \mathcal{V}}} \max _{\pi} \frac{|\tilde{\lambda} \psi-\tilde{\vartheta} \phi|}{1-\tilde{\vartheta} \phi}\right]\right)
$$

Let us re-write the first term on the right side of (18). The idea is to use Lemma 1 with $\mathcal{V}=\mathcal{C}$ as an ultimate upper bound to the RA error. Then, subsequently we improve by using the full potential of $\mathcal{V}$. We first exploit that whenever $\max _{\pi} \psi \geq 1-\epsilon$, for some $\epsilon \leq \frac{1}{2}$, then by Lemma 1 the error can be uniformly bounded from above by $\frac{\tilde{\lambda} \epsilon}{1-\tilde{\lambda} \epsilon}=\frac{\lambda^{2} \epsilon}{1+\lambda^{2}(1-\epsilon)} \leq \frac{\lambda^{2} \epsilon}{1+\lambda^{2} \epsilon}$, and since clearly $\max _{\pi} \phi \geq \frac{1}{n_{t}}$ and $1-\epsilon \leq \frac{\epsilon}{n_{t}-1}$ for $\epsilon \leq 1-\frac{1}{n_{t}}$ we have for $\max _{\pi} \psi \geq \max (0,1-\epsilon) \frac{\lambda^{2} \epsilon}{1+\lambda^{2} \epsilon} \leq \frac{\lambda^{2} \epsilon}{1+\lambda^{2} \frac{\epsilon}{n_{t}-1}}=\frac{\tilde{\lambda} \epsilon}{1+\tilde{\lambda}\left(\frac{\epsilon}{n_{t}-1}-1\right)}$, for any $\epsilon>0$ (even that for $\epsilon>1$ ). On the other hand, we have $\max _{\pi} \psi<\max (0,1-\epsilon) \frac{|\tilde{\lambda} \psi-\tilde{\vartheta} \phi|}{1-\tilde{\lambda} \psi} \leq \frac{|\tilde{\lambda} \psi-\tilde{\vartheta} \phi|}{1-\tilde{\lambda}+\tilde{\lambda} \epsilon} \leq \frac{|\tilde{\lambda} \psi-\tilde{\vartheta} \phi|}{1+\tilde{\lambda}\left(\frac{\epsilon}{n_{t}-1}-1\right)}$. Hence, we can write for some pair $(\boldsymbol{h}, \boldsymbol{\nu}): \frac{|\tilde{\lambda} \psi-\tilde{\vartheta} \phi|}{1-\tilde{\lambda} \psi} \leq \frac{\max \{\tilde{\lambda} \epsilon,|\tilde{\lambda} \psi-\tilde{\vartheta} \phi|\}}{1+\tilde{\lambda}\left(\frac{\epsilon}{n_{t}-1}-1\right)}$ and setting $\tilde{\lambda} \epsilon=\min _{\substack{1>\tilde{\vartheta}>\mathcal{\nu} \\ \boldsymbol{\nu} \in \mathcal{V}}} \max _{\pi}|\tilde{\lambda} \psi-\tilde{\vartheta} \phi|=$ $\left|\tilde{\lambda} \psi^{*}-\tilde{\vartheta}^{*} \phi^{*}\right|$, where $\phi^{*}=\left|\left\langle\boldsymbol{\nu}^{*}, \boldsymbol{w}_{\pi^{*}(m)}\right\rangle\right|^{2}$ and $\psi^{*}=\left|\left\langle\boldsymbol{h}, \boldsymbol{w}_{\pi^{*}(m)}\right\rangle\right|^{2}$ with respect to maximizing mapping $\pi^{*}$ and minimizing arguments $\left(\boldsymbol{\nu}^{*}, \tilde{\vartheta}^{*}\right)$. This yields $\min _{\substack{1>\tilde{\vartheta}>0 \\ \nu \in \mathcal{V}}} \max _{\pi} \frac{|\tilde{\lambda} \psi-\tilde{\vartheta} \phi|}{1-\tilde{\lambda} \psi} \leq \frac{\left|\tilde{\lambda} \psi^{*}-\tilde{\vartheta}^{*} \phi^{*}\right|}{1-\tilde{\lambda}+\frac{1}{n_{t}-1}\left|\tilde{\lambda} \psi^{*}-\tilde{\vartheta}{ }^{*} \phi^{*}\right|}$. Equivalently, for the second term on the right side of $(18)$ we have $\frac{|\tilde{\lambda} \psi-\tilde{\vartheta} \phi|}{1-\tilde{\vartheta} \phi} \leq \frac{\max \{\tilde{\lambda} \epsilon,|\tilde{\lambda} \psi-\tilde{\vartheta} \phi|\}}{1-\tilde{\lambda} \psi+\tilde{\lambda} \psi-\tilde{\vartheta} \phi} \leq \frac{\max \{\tilde{\lambda} \epsilon,|\tilde{\lambda} \psi-\tilde{\vartheta} \phi|\}}{1+\tilde{\lambda}_{m}\left(\frac{\max \{\epsilon-|\tilde{\lambda} \psi-\tilde{\vartheta} \phi|, 0\}}{n_{t}-1}-1\right)}$. Setting $\epsilon=\left(1+\epsilon^{\prime}\right)\left|\tilde{\lambda} \psi^{*}-\tilde{\vartheta}^{*} \phi^{*}\right|, \epsilon^{\prime}>0$, since the error term is still increasing in $\epsilon$, yields $\min _{\substack{1>\tilde{\vartheta}>0 \\ \nu \in \mathcal{V}}} \max _{\pi} \frac{|\tilde{\lambda} \psi-\tilde{\vartheta} \phi|}{1-\tilde{\vartheta} \phi} \leq \frac{\left(1+\epsilon^{\prime}\right)\left|\tilde{\lambda} \psi^{*}-\tilde{\vartheta}^{*} \phi^{*}\right|}{1-\tilde{\lambda}+\frac{\epsilon^{\prime}}{n_{t}-1}\left|\tilde{\lambda} \psi^{*}-\tilde{\vartheta} \phi^{*}\right|}$, Finally, expanding the fraction with $(1-\tilde{\lambda})$ and applying Jensen's inequality again proves the claim.

\section{APPENDIX C}

\section{PROOF OF LEMMA 3}

Proof: Consider an arbitrary but fixed user $m \in \mathcal{U}$ and, without loss of generality, assume the transmit codebook $\mathcal{C}$ is given by the standard ONB. Define the vector

$\boldsymbol{\psi}_{m}=\left(\left|\left\langle\boldsymbol{h}_{m}, \boldsymbol{w}_{\pi(1)}\right\rangle\right|^{2}, \ldots,\left|\left\langle\boldsymbol{h}_{m}, \boldsymbol{w}_{\pi\left(n_{t}\right)}\right\rangle\right|^{2}\right)$. Since, $\left\|\boldsymbol{h}_{m}\right\|=1$ from the Parseval's identity follows that $\left\|\boldsymbol{\psi}_{m}\right\|_{1}=1$ and, therefore, any $\boldsymbol{\psi}_{m}$ corresponds to a point on the $d:=n_{t}-1$ dimensional simplex defined by $\mathcal{K}_{d}=\left\{\boldsymbol{x} \in \mathbb{R}^{d+1}: x_{i}>0, i=\right.$ $1, \ldots, n_{t}$ and $\left.\|\boldsymbol{x}\|_{1}=1\right\}$, which has edge length $\sqrt{2}$. Similarly, each element of the feedback codebook $\boldsymbol{\nu} \in \mathcal{V}$ defines a point $\boldsymbol{q} \in \mathcal{K}_{d}$ on the $d$-simplex, collected in the set $\mathcal{Q} \subset \mathcal{K}_{d}$, with $|\mathcal{Q}|=|\mathcal{V}|=2^{B}$. Setting $\tilde{\vartheta}_{m}=\tilde{\lambda}_{m}$ the function $D_{m}(B)$ can be bounded from above by

$$
D_{m}(B) \leq \mathbb{E}_{H}\left[\lambda_{m}^{2}\right] \max _{\boldsymbol{x} \in \mathcal{K}_{d}} \min _{\boldsymbol{q} \in \mathcal{Q}}\|\boldsymbol{x}-\boldsymbol{q}\|_{\infty}
$$

Now we show that $\delta:=\max _{\boldsymbol{x} \in \mathcal{K}_{d}} \min _{\boldsymbol{q} \in \mathcal{Q}}\|\boldsymbol{x}-\boldsymbol{q}\|_{\infty}$ can be bounded from above by $c\left(n_{t}\right) 2^{-\frac{B}{\left(n_{t}-1\right)}}$, with $c\left(n_{t}\right)$ a constant. Consider the cubes $\mathcal{B}_{\infty}^{n_{t}}(\boldsymbol{y} ; \delta):=\left\{\boldsymbol{x} \in \mathbb{R}^{n_{t}}:\|\boldsymbol{y}-\boldsymbol{x}\|_{\infty} \leq \delta\right\}$. If $\boldsymbol{y} \in \mathcal{K}_{d}$, then the intersection of the centered cubes $\mathcal{B}_{\infty}^{n_{t}}(\boldsymbol{y} ; \delta)$ with $\mathcal{K}_{d}$ is a polytope with $2 d$ facets. Let $\mathcal{B}_{2}^{d}(\delta):=\left\{\boldsymbol{x} \in \mathbb{R}^{d}:\|\boldsymbol{x}\|_{2} \leq \delta\right\}$ be the balls with radius $\delta$ that are inscribed in this polytopes. Hence, to upperbound the number of centered cubes $\mathcal{B}_{\infty}^{n_{t}}(\boldsymbol{y} ; \delta)$, with $\boldsymbol{y} \in \mathcal{K}_{d}$, required to cover the simplex $\mathcal{K}_{d}$ we 
need to compute the number of balls $\mathcal{B}_{2}^{d}(\delta)$ required to cover $\mathcal{K}_{d}$. Let the number of balls $\mathcal{B}_{2}^{d}(\delta)$ required to cover the simplex $\mathcal{K}^{d}$ be given by the covering number $N\left(\mathcal{K}_{d}, \mathcal{B}_{2}^{d}(\delta)\right)$. The covering number $N(\mathcal{A}, \mathcal{B})$ is defined as the number of convex bodies $\mathcal{B}$ in $\mathbb{R}^{d}$ required to cover a convex body $\mathcal{A}$ in $\mathbb{R}^{d}$. Using the Rogers-Zong Lemma [25] the covering number can be bounded from above by

$$
N(\mathcal{A}, \mathcal{B}) \leq \Theta(\mathcal{B}) \frac{\operatorname{vol}(\mathcal{A}-\mathcal{B})}{\operatorname{vol}(\mathcal{B})}
$$

where $\operatorname{vol}(\cdot)$ is a function that computes the volume and $\Theta(\mathcal{B}) \geq 1$ is the covering density of $\mathcal{B}$; if $\mathbb{R}^{d}$ can be tiled by translates of $\mathcal{B}$ then $\Theta(\mathcal{B})=1$; if the covering has some overlap then $\Theta(\mathcal{B})>1$. Now we can use the Rogers-Shephard inequality [26], which states that

$$
\operatorname{vol}(\mathcal{A}-\mathcal{B}) \operatorname{vol}(\mathcal{A} \cap \mathcal{B}) \leq\left(\begin{array}{c}
2 d \\
d
\end{array}\right) \operatorname{vol}(\mathcal{A}) \operatorname{vol}(\mathcal{B})
$$

Assuming that $\operatorname{vol}(\mathcal{A} \cap \mathcal{B})=\operatorname{vol}(\mathcal{B})$ we get from 19 and 20 that the covering number $N(\mathcal{A}, \mathcal{B})$ is upper bounded by

$$
N(\mathcal{A}, \mathcal{B}) \leq \Theta(\mathcal{B})\left(\begin{array}{c}
2 d \\
d
\end{array}\right) \frac{\operatorname{vol}(\mathcal{A})}{\operatorname{vol}(\mathcal{B})}
$$

Now we can apply this bound to our problem. The volumes of the $d$-simplex $\mathcal{K}_{d}$ and the balls $\mathcal{B}_{2}^{d}(\delta)$ are vol $\left(\mathcal{K}_{d}\right)=\frac{\sqrt{d+1}}{d !}$ and $\operatorname{vol}\left(\mathcal{B}_{2}^{d}(\delta)\right)=\frac{\pi^{d / 2}}{\Gamma(1+d / 2)} \delta^{d}$, where $\Gamma(\cdot)$ is the gamma function. Hence, the covering number can be bounded from above by

$$
N\left(\mathcal{K}_{d}, \mathcal{B}_{2}^{d}(\delta)\right)=N\left(\frac{1}{\delta} \mathcal{K}_{d}, \mathcal{B}_{2}^{d}(1)\right) \leq \Theta\left(\mathcal{B}_{2}^{d}(1)\right)\left(\begin{array}{c}
2 d \\
d
\end{array}\right) \frac{\Gamma(1+d / 2) \sqrt{d+1}}{d ! \pi^{d / 2}} \cdot \frac{1}{\delta^{d}} .
$$

Solving for $\delta$ and using $2^{B} \leq N\left(\mathcal{K}_{d}, \mathcal{B}_{2}^{d}(\delta)\right)=$ we get

$$
\delta \leq\left(\Theta\left(\mathcal{B}_{2}^{d}\right)\left(\begin{array}{c}
2 n_{t}-2 \\
n_{t}-1
\end{array}\right) \frac{\Gamma\left(1+\frac{n_{t}-1}{2}\right) \sqrt{n_{t}}}{\left(n_{t}-1\right) ! \pi^{\frac{n_{t}-1}{2}}}\right)^{\frac{1}{n_{t}-1}} 2^{-\frac{B}{n_{t}-1}} .
$$

Finally, for (21) to be valid we need to ensure that $\operatorname{vol}\left(\mathcal{K}_{d} \cap \mathcal{B}_{2}^{d}(\delta)\right)=\operatorname{vol}\left(\mathcal{B}_{2}^{d}(\delta)\right)$ or, in other words, $\delta$ is smaller than the inradius of the inscribed circle of the simplex. According to Klamkin [27] for a regular simplex the inradius equals the circumradius divided by $n_{t}-1$. The circumradius is easily shown by the volume ratio and Stirlings formula to be greater than $\sqrt{n_{t}-1}$.

\section{APPENDIX D}

\section{ProOF OF THEOREM 1}

Proof: Similarly to Lemma1 the terms of the sum in (6) can be bounded from above. We use again $\phi_{m l}:=\left|\left\langle\boldsymbol{\nu}_{m}, \boldsymbol{w}_{\pi(l)}\right\rangle\right|^{2}$ and $\psi_{m l}:=\left|\left\langle\boldsymbol{h}_{m}, \boldsymbol{w}_{\pi(l)}\right\rangle\right|^{2}$ without writing explicitly the dependency on the mapping $\pi$ and get

$$
\begin{aligned}
r_{m}\left(\pi, \mathcal{S}, \lambda_{m} \boldsymbol{h}_{m}\right)-r_{m}\left(\pi, \mathcal{S}, \vartheta_{m} \boldsymbol{\nu}_{m}\right) & =\log \left(\frac{\frac{|\mathcal{S}|}{n_{t}}+\lambda_{m}^{2} \sum_{l \in \mathcal{S}} \psi_{m l}}{\frac{|\mathcal{S}|}{n_{t}}+\lambda_{m}^{2} \sum_{l \in \mathcal{S} \backslash\{m\}} \psi_{m l}}\right)-\log \left(\frac{\frac{|\mathcal{S}|}{n_{t}}+\vartheta_{m}^{2} \sum_{l \in \mathcal{S}} \phi_{m l}}{\frac{\mathcal{S} \mid}{n_{t}}+\vartheta_{m}^{2} \sum_{l \in \mathcal{S} \backslash\{m\}} \phi_{m l}}\right) \\
& =\log \left(\frac{\frac{|\mathcal{S}|}{n_{t}}+\lambda_{m}^{2} \sum_{l \in \mathcal{S}} \psi_{m l}}{\frac{|\mathcal{S}|}{n_{t}}+\vartheta_{m}^{2} \sum_{l \in \mathcal{S}} \phi_{m l}}\right)+\log \left(\frac{\frac{|\mathcal{S}|}{n_{t}}+\vartheta_{m}^{2} \sum_{l \in \mathcal{S} \backslash\{m\}} \phi_{m l}}{\frac{|\mathcal{S}|}{n_{t}}+\lambda_{m}^{2} \sum_{l \in \mathcal{S} \backslash\{m\}} \psi_{m l}}\right) .
\end{aligned}
$$

Setting $\vartheta_{m}^{2}=\lambda_{m}^{2}$ we get the inequality chain

$$
\begin{aligned}
r_{m}\left(\pi, \mathcal{S}, \lambda_{m} \boldsymbol{h}_{m}\right)-r_{m}\left(\pi, \mathcal{S}, \lambda_{m} \boldsymbol{\nu}_{m}\right) & =\log \left(1+\frac{n_{t} \lambda_{m}^{2}}{|\mathcal{S}|} \frac{\sum_{l \in \mathcal{S}} \psi_{m l}-\phi_{m l}}{1+\lambda_{m}^{2} \sum_{l \in \mathcal{S}} \phi_{m l}}\right)+\log \left(1+\frac{n_{t} \lambda_{m}^{2}}{|\mathcal{S}|} \frac{\sum_{l \in \mathcal{S} \backslash\{m\}} \phi_{m l}-\psi_{m l}}{1+\lambda_{m}^{2} \sum_{l \in \mathcal{S} \backslash\{m\}} \psi_{m l}}\right) \\
& \leq \log \left(1+\frac{n_{t} \lambda_{m}^{2}}{|\mathcal{S}|}\left|\sum_{l \in \mathcal{S}} \psi_{m l}-\phi_{m l}\right|\right)+\log \left(1+\frac{n_{t} \lambda_{m}^{2} \mid}{|\mathcal{S}|} \sum_{l \in \mathcal{S} \backslash\{m\}} \psi_{m l}-\phi_{m l} \mid\right) \\
& \leq \log \left(1+\frac{n_{t} \lambda_{m}^{2}}{|\mathcal{S}|}|\mathcal{S}| \max _{\pi}\left|\psi_{m m}-\phi_{m m}\right|\right)+\log \left(1+\frac{n_{t} \lambda_{m}^{2}}{|\mathcal{S}|}|\mathcal{S} \backslash\{m\}| \cdot \max _{\pi}\left|\psi_{m m}-\phi_{m m}\right|\right) \\
& \leq 2 \log \left(1+n_{t} \lambda_{m}^{2} \max _{\pi}\left|\psi_{m m}-\phi_{m m}\right|\right) \\
& =2 \log \left(1+\frac{P\left\|\hat{\boldsymbol{h}}_{m}\right\|_{2}^{2}}{\sigma^{2}} \max _{\pi}\left|\psi_{m m}-\phi_{m m}\right|\right)
\end{aligned}
$$


The lower bound on $-\left(r_{m}\left(\pi, \mathcal{S}, \lambda_{m} \boldsymbol{h}_{m}\right)-r_{m}\left(\pi, \mathcal{S}, \vartheta_{m} \boldsymbol{\nu}_{m}\right)\right)$ can be obtained in a similar manner. Taking expectations and using Jensen's inequality we obtain

$$
\mathbb{E}_{H}\left[r_{m}\left(\pi, \mathcal{S}, \lambda_{m} \boldsymbol{h}_{m}\right)-r_{m}\left(\pi, \mathcal{S}, \lambda_{m} \boldsymbol{\nu}_{m}\right)\right] \leq 2 \log \left(1+\mathbb{E}_{\boldsymbol{H}}\left[\frac{P\left\|\hat{\boldsymbol{h}}_{m}\right\|_{2}^{2}}{\sigma^{2}} \max _{\pi}\left|\psi_{m m}-\phi_{m m}\right|\right]\right) .
$$

Since $\max _{\pi}\left|\psi_{m m}-\phi_{m m}\right|$ depends only on the channel directions $\boldsymbol{h}_{m}$, it is independent of the channel magnitude $\left\|\hat{\boldsymbol{h}}_{m}\right\|_{2}$.

$$
\mathbb{E}_{H}\left[r_{m}\left(\pi, \mathcal{S}, \lambda_{m} \boldsymbol{h}_{m}\right)-r_{m}\left(\pi, \mathcal{S}, \lambda_{m} \boldsymbol{\nu}_{m}\right)\right] \leq 2 \log \left(1+\frac{P n_{t}}{\sigma^{2}} \mathbb{E}_{H}\left[\max _{\pi}\left|\psi_{m m}-\phi_{m m}\right|\right]\right) .
$$

Using the RA scheme and (6) yields the result.

\section{REFERENCES}

[1] D. Love, R. Heath, V. N. Lau, D. Gesbert, B. Rao, and M. Andrews, "An overview of limited feedback in wireless communication systems," IEEE Journal on Selected Areas in Communications, vol. 26, no. 8, pp. 1341-1365, Oct. 2008.

[2] K. Kiran Mukkavilli, A. Sabharwal, E. Erkip, and B. Aazhang, "On beamforming with finite rate feedback in multiple-antenna systems," IEEE Transactions on Information Theory, vol. 49, no. 10, pp. 2562-2579, Oct. 2003.

[3] C. Au-Yeung and D. Love, "On the performance of random vector quantization limited feedback beamforming in a MISO system," IEEE Transactions on Wireless Communications, vol. 6, no. 2, pp. 458-462, Feb. 2007.

[4] W. Santipach and M. L. Honig, "Capacity of a multiple-antenna fading channel with a quantized precoding matrix," IEEE Transactions on Information Theory, vol. 55, no. 3, pp. 1218-1234, Mar. 2009.

[5] N. Jindal, "MIMO broadcast channels with finite-rate feedback," IEEE Transactions on Information Theory, vol. 52, no. 11, pp. 5045-5060, Nov. 2006.

[6] M. Kountouris, R. de Francisco, D. Gesbert, D. T. M. Slock, and T. Sälzer, "Efficient metrics for scheduling in MIMO broadcast channels with limited feedback," in IEEE International Conference on Acoustics, Speech and Signal Processing, vol. 3, Apr. 2007, pp. III-109-III-112.

[7] M. Trivellato, F. Boccardi, and H. Huang, "On transceiver design and channel quantization for downlink multiuser MIMO systems with limited feedback," IEEE Journal on Selected Areas in Communications, vol. 26, no. 8, pp. 1494-1504, Oct. 2008.

[8] P. Ding, D. J. Love, and M. D. Zoltowski, "Multiple antenna broadcast channels with shape feedback and limited feedback," IEEE Transactions on Signal Processing, vol. 55, no. 7, pp. 3417-3428, Jul. 2007.

[9] K. Huang, J. Heath, and J. G. Andrews, "Space division multiple access with a sum feedback rate constraint," IEEE Transactions on Signal Processing, vol. 55, no. 7, pp. 3879-3891, Jul. 2007.

[10] R. Samsung, "Downlink MIMO for EUTRA," 3GPP TSG RAN WG1 Meeting, Feb. 2006.

[11] C. Zhang, W. Xu, and M. Chen, "Multi-mode transmission for MIMO broadcast channels with PU2RC," in 2009 15th Asia-Pacific Conference on Communications. IEEE, Oct. 2009, pp. 293-296.

[12] J. Zhang, M. Kountouris, J. Andrews, and R. Heath, "Multi-mode transmission for the MIMO broadcast channel with imperfect channel state information," IEEE Transactions on Communications, vol. 59, no. 3, pp. 803-814, Mar. 2011.

[13] G. Dimic and N. Sidiropoulos, "On downlink beamforming with greedy user selection: Performance analysis and a simple new algorithm," IEEE Transactions on Signal Processing, vol. 53, no. 10, pp. 3857-3868, Oct. 2005.

[14] J. Schreck, P. Jung, G. Wunder, M. Ohm, and H.-P. Mayer, "Limited feedback in multiuser MIMO OFDM systems based on rate approximation," in IEEE Global Telecommunications Conference, Nov. 2009.

[15] M. Sharif and B. Hassibi, "On the capacity of MIMO broadcast channels with partial side information," IEEE Transactions on Information Theory, vol. 51, no. 2, pp. 506-522, Feb. 2005.

[16] T. Yoo, N. Jindal, and A. Goldsmith, "Multi-antenna downlink channels with limited feedback and user selection," IEEE Journal on Selected Areas in Communications, vol. 25, no. 7, pp. 1478-1491, Sep. 2007.

[17] K. Huang, J. G. Andrews, and R. Heath, "Performance of orthogonal beamforming for SDMA with limited feedback," IEEE Transactions on Vehicular Technology, vol. 58, no. 1, pp. 152-164, 2009.

[18] G. Wunder and J. Schreck, "A robust and efficient transmission technique for the lte downlink," in 44th Annual Asilomar Conference on Signals, Systems, and Computers, Dec. 2010.

[19] R. de Francisco, M. Kountouris, D. T. M. Slock, and D. Gesbert, "Orthogonal linear beamforming in MIMO broadcast channels," in 2007 IEEE Wireless Communications and Networking Conference, Mar. 2007, pp. 1210-1215.

[20] G. Wunder, J. Schreck, P. Jung, H. Huang, and R. Valenzuela, "Rate approximation: A new paradigm for multiuser MIMO downlink communications," in IEEE International Conference on Communications (ICC), May 2010.

[21] K. Böröczky, Finite packing and covering. Cambridge University Press, 2004.

[22] 3GPP TS 36.201, "LTE Physical Layer - General Description," 2009.

[23] D. S. Baum, J. Salo, M. Milojevic, P. Kyösti, and J. Hansen, "MATLAB implementation of the interim channel model for beyond-3G systems (SCME)," 2005.

[24] M. Trivellato, F. Boccardi, and F. Tosato, "User selection schemes for MIMO broadcast channels with limited feedback," IEEE 65th Vehicular Technology Conference, pp. 2089-2093, Apr. 2007.

[25] C. A. Rogers and C. Zong, "Covering convex bodies by translates of convex bodies," Mathematika, vol. 44, no. 01, pp. $215-218$, Jun. 1997.

[26] C. Rogers and G. Shephard, The difference body of a convex body. Springer, 1957, vol. 8, no. 3.

[27] M. S. Klamkin and G. A. Tsintsifas, The Circumradius-Inradius Inequality for a Simplex. Mathematical Association of America, Jan. 1979, vol. 52, no. 1 . 
Gerhard Wunder (M'05) studied electrical engineering at the University of Hannover, Germany, and the Technische Universität (TU) Berlin, Germany, and received his graduate degree in electrical engineering (Dipl.-Ing.) with highest honors in 1999 and the $\mathrm{PhD}$ degree (Dr.-Ing.) in communication engineering on the peak-to-average power ratio (PAPR) problem in OFDM with distinction (summa cum laude) in 2003 from TU Berlin. In 2007, he also received the habilitation degree (venia legendi) and became a

PLACE Privatdozent at the TU Berlin in the field of detection/estimation theory, stochastic processes and information theory. Since 2003 PHOTO he is heading a research group at the Fraunhofer Lab for Mobile Communications (FhG-MCI) working in close collaboration with industry on theoretical and practical problems in wireless communication networks particularly in the field of LTE-A systems. He is a recipient of research fellowships from the German national research foundation.

In 2000 and 2005, he was a visiting professor at the Georgia Institute of Technology (Prof. Jayant) in Atlanta (USA, GA), and the Stanford University (Prof. Paulraj) in Palo Alto/USA (CA). In 2009 he was a consultant at Alcatel-Lucent Bell Labs (USA, NJ), both in Murray Hill and Crawford Hill. He was a general co-chair of the 2009 International ITG Workshop on Smart Antennas (WSA 2009) and a lead guest editor in 2011 for a special issue of the Journal of Advances on Signal Processing regarding the PAPR problem of the European Association for Signal Processing. Since 2011, he is also an editor for the IEEE Transactions on Wireless Communications (TWireless) in the area of Wireless Communications Theory and Systems (WCTS). In 2011 Dr. Wunder received the best paper award for outstanding scientific publication in the field of communication engineering by the German communication engineering society (ITG Price 2011).

PLACE

PHOTO

HERE
Jan Schreck (Member IEEE) received the Dipl.-Math. in 2006 from the University of Applied Science Berlin, Germany, in cooperation with the Weierstrass Institute for Applied Analysis and Stochastics Berlin, Germany. Since 2006 he has been with the Fraunhofer German-Sino Lab for Mobile Communications and since 2011 he is with the Technische Universität Berlin, Germany, where he is currently working toward the Ph.D. degree. His research interests include information theory and signal processing.
PLACE

PHOTO

HERE
Peter Jung (Member IEEE, Member VDE/ITG) received the Dipl.-Phys. in high energy physics in 2000 from Humboldt University, Berlin, Germany, in cooperation with DESY Hamburg. Since 2001 he has been with the Department of Broadband Mobile Communication Networks, Fraunhofer Institute for Telecommunications, Heinrich-Hertz-Institut (HHI) and since 2004 with Fraunhofer GermanSino Lab for Mobile Communications. He received the Dr.-rer.nat (Ph.D.) degree in 2007 (on Weyl-Heisenberg representations in communication theory) at the Technical University of Berlin (TUB), Germany. P. Jung is currently working under DFG grants JU 27951-1\&2 at the Technical University in Berlin, Germany (TUB) and Technical University in Munich, Germany (TUM) in the field information theory and signal processing. His current research interests are in the area time-frequency analysis, compressed sensing, dimension reduction and randomized algorithms. He is giving lectures in compressed sensing and estimation theory. 